\title{
Semi-Empirical Model of the Ensemble-Averaged Differential Mueller Matrix for Microwave Backscattering From Bare Soil Surfaces
}

\author{
Yisok Oh, Member, IEEE, Kamal Sarabandi, Fellow, IEEE, and Fawwaz T. Ulaby, Fellow, IEEE
}

\begin{abstract}
A semi-empirical model of the ensemble-averaged differential Mueller matrix for microwave backscattering from bare soil surfaces is presented. Based on existing scattering models and data sets measured by polarimetric scatterometers and the JPL AirSAR, the parameters of the co-polarized phase-difference probability density function, namely the degree of correlation $\alpha$ and the co-polarized phase-difference $\varsigma$, in addition to the backscattering coefficients $\sigma_{v v}^{0}, \sigma_{h h}^{0}$ and $\sigma_{v h}^{0}$, are modeled empirically in terms of the volumetric soil moisture content $m_{v}$ and the surface roughness parameters $k s$ and $k l$, where $k=2 \pi f / c, s$ is the rms height and $l$ is the correlation length. Consequently, the ensemble-averaged differential Mueller matrix (or the differential Stokes scattering operator) is specified completely by $\sigma_{v v}^{0}, \sigma_{h h}^{0}$, $\sigma_{v h}^{0}, \alpha$, and $\zeta$
\end{abstract}

Index Terms-Bare soil surfaces, differential Mueller matrix, microwave backscattering, semi-empirical model.

\section{INTRODUCTION}

$\mathbf{T}$ HERE is a strong need for a good polarimetric scattering model for backscattering from bare soil surfaces for various reasons. One of the reasons is to use the phase-difference statistics, in addition to the backscattering coefficients, for retrieving the soil moisture and the surface roughness parameters from synthetic aperture radar (SAR) data. The other reason for the need to generate a good model for the ensemble-averaged differential Mueller matrix (or the Stokes scattering operator) for backscatter by bare soil surfaces is that it is a prerequisite for the development of a scattering model for vegetation-covered surfaces. The polarimetric scattering model can also be used to synthesize the polarization response for any possible combination of transmit and receive antenna polarizations.

The small perturbation method (SPM), the physical optics (PO) model, the geometrical optics (GO) model [1] and the integral equation method (IEM)[2]-[5] are commonly used for predicting the backscattering coefficients of rough surfaces. Empirical models based on polarimetric measurements have also been reported [6], [7], but they have dealt with only the magnitude of the backscattering response; i.e., the backscattering coefficients, $\sigma_{v v}^{0}, \sigma_{h h}^{0}$, and $\sigma_{v h}^{0}$. Experimental data acquired by

Manuscript received April 24, 2001; revised April 9, 2002. This work was supported by the Agency for Defense Development of Korea and the Brain Korea 21 Project.

Y. Oh is with the Department of Electronics and Electrical Engineering, Hong-ik University, Seoul, Korea.

K. Sarabandi and F. T. Ulaby are with the Radiation Laboratory, Department of Electrical Engineering and Computer Science, University of Michigan, Ann Arbor, MI 48109 USA.

Publisher Item Identifier 10.1109/TGRS.2002.800232. coherent polarimetric SAR systems and by polarimetric scatterometer systems have shown that the probability density function (PDF) of the co-polarized phase angle $\phi_{c}=\phi_{h h}-\phi_{v v}$, as well as the backscattering coefficients, are strongly dependent upon the incidence angle, the wavelength, the soil moisture content and surface roughness. In contrast, the cross-polarized phase angle $\phi_{x}=\phi_{v h}-\phi_{v v}=\phi_{h v}-\phi_{v v}$ is uniformly distributed over $[0,2 \pi]$ and therefore contains no target-specific information [8]-[10]. The PDF of $\phi_{c}$ is characterized completely by two parameters, namely the degree of correlation $\alpha$ and the co-polarized phase-difference $\zeta[9]$.

Unlike the backscattering coefficients of bare soil surfaces, no theoretical models currently exist for the parameters $\alpha$ and $\zeta$, even though many experimental observations have been reported [8]-[11]. An attempt for modeling empirically the phase parameters $\alpha$ and $\zeta$ had been tried based on a data set obtained by a ground-based scatterometer [12], [13]. The goal of this study is to improve the empirical models for the parameters $\alpha$ and $\zeta$, as well as $\sigma_{v v}^{0}, \sigma_{h h}^{0}$ and $\sigma_{v h}^{0}$, based on an extensive database obtained by the JPL airborne SAR system and groundbased scatterometers, thereby providing a complete model for all of the ensemble-averaged differential Mueller matrix elements.

The backscattering coefficient of a distributed target can be computed from the following polarization synthesis equation [14]:

$$
\sigma_{r t}^{0}\left(\psi_{r}, \chi_{r} ; \psi_{t}, \chi_{t}\right)=4 \pi \bar{A}^{r} \cdot \overline{\bar{Q}} \overline{\bar{M}}^{0} \bar{A}^{t}
$$

where $\psi$ is the rotation angle, $\chi$ is the ellipticity angle of the polarization ellipse, $\bar{A}^{r}$ and $\bar{A}^{t}$ are the normalized modified Stokes vectors for the receiving and transmitting antennas. We note that $\bar{Q} \bar{M}^{0}$ is the modified differential Stokes scattering operator [14], where $\bar{M}^{0}$ is the ensemble-averaged differential Mueller matrix [15].

From a measurement standpoint, if we assume that the differential scattering matrix is uniform across the area illuminated by the main beam of the antenna, the differential Mueller matrix is then obtained from $\bar{M}^{0} \approx \bar{M} / A_{i l l}$, where $A_{\underline{i} l l}$ is the illuminated area (or the illumination integral) and $\bar{M}$ is the measured ensemble-averaged modified Mueller matrix. In practice, the uniformity assumption is seldom applicable, thereby necessitating the application of an elaborate calibration procedure as described in [15].

Analysis of polarimetric data for various types of distributed targets, such as soil surfaces, indicates that the correlation 


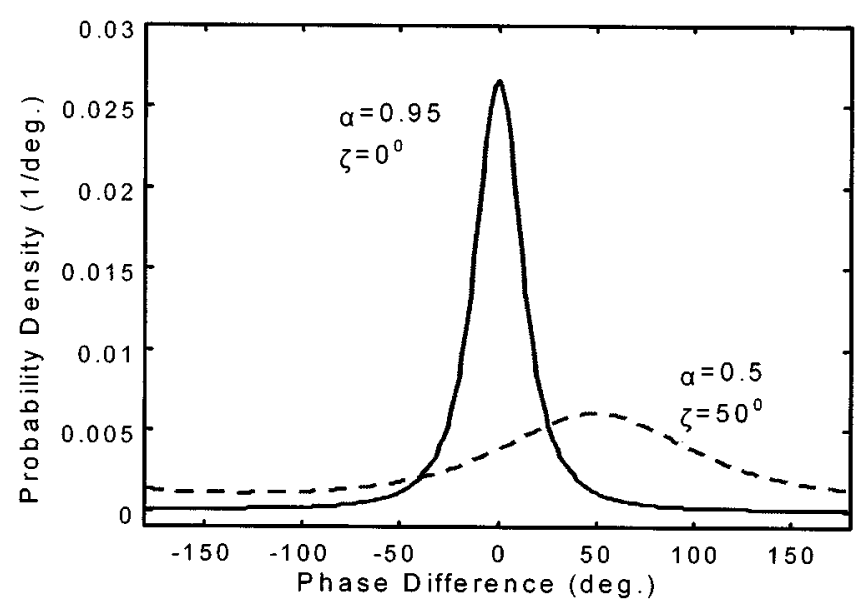

Fig. 1. Phase-difference PDFs with different values of $\alpha$ and $\zeta$.

between the two co-polarized scattering amplitudes $S_{v v}^{0} S_{h h}^{0 *}$ is quite significant, while that between the co- and cross-polarized scattering amplitudes $S_{h v}^{0} S_{h h}^{0 *}, S_{v v}^{0} S_{v h}^{0 *}, S_{v v}^{0} S_{h v}^{0 *}$ or $S_{v h}^{0} S_{h h}^{0 *}$ is very weak [8]-[11], [16]. According to the reciprocity relation, the cross-polarized scattering amplitudes are identical in the backscattering direction, namely $S_{v h}^{0}=S_{h v}^{0}$. Therefore, the differential Mueller matrix can be approximated as (2), shown at the bottom of the page.

The PDF of the co-polarized phase angle $\phi_{c}=\phi_{h h}-\phi_{v v}$ was derived in [9] as follows:

$$
\begin{aligned}
f_{\Phi}\left(\phi_{C}\right) & =\frac{1-\alpha^{2}}{2 \pi\left(1-X^{2}\right)} \\
& \cdot\left\{1+\frac{X}{\sqrt{1-X^{2}}}\left[\frac{\pi}{2}+\tan ^{-1}\left(\frac{X}{\sqrt{1-X^{2}}}\right)\right]\right\}
\end{aligned}
$$

where $X=\alpha \cos \left(\phi_{c}-\zeta\right)$, the parameter $\alpha$, referred to as the degree of correlation, is a measure of the width of the PDF and the parameter $\zeta$, referred to as the mean value of the co-polarized phase-difference [9], is the value of $\phi_{c}$ at which the PDF has a maximum. These two parameters completely specify $f_{\Phi}\left(\phi_{c}\right)$. Fig. 1 shows plots of the PDF for two different values of $\alpha$ and $\zeta$. The PDF is approximately Gaussian in shape with a circular nature over $\left[-180^{\circ}, 180^{\circ}\right]$. Fig. 2 shows the relation between the parameter $\alpha$ and the standard deviation of $\phi_{c}, \sigma_{\phi_{c}}$, of the corresponding Gaussian PDF with $\zeta=0$. The degree of correlation $\alpha$ is directly related to the standard deviation of $\phi_{c}, \sigma_{\phi_{c}}$ and the co-polarized phase-difference $\zeta$ is equivalent to the mean of $\phi_{c}, \bar{\phi}_{c}$.

The ensemble-averaged terms of the co-polarized scattering amplitudes in the differential Mueller matrix elements were de-

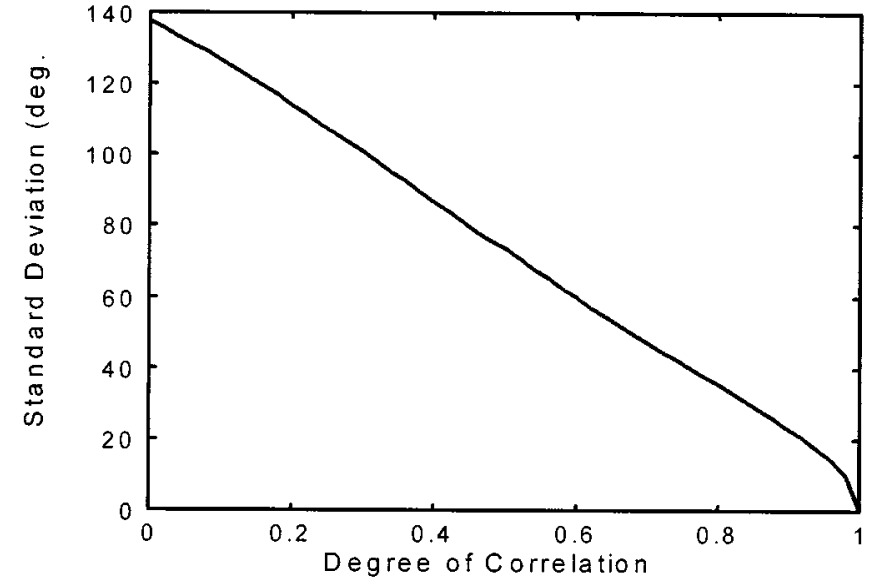

Fig. 2. Relation between the phase-difference standard deviation $\alpha_{\phi_{c}}$ and the degree of correlation $\alpha$.

rived in [9], [10] using the parameters $\alpha$ and $\zeta$ as follows:

$$
\begin{aligned}
\left\langle\operatorname{Re}\left(S_{v v^{0}}^{0} S_{h h}^{0 *}\right)\right\rangle & =\alpha \cos \zeta \sqrt{M_{11}^{0} M_{22}^{0}} \\
\left\langle\operatorname{Im}\left(S_{v v^{0}}^{0} S_{h h^{*}}^{0}\right)\right\rangle & =\alpha \sin \zeta \sqrt{M_{11}^{0} M_{22}^{0}} .
\end{aligned}
$$

The ensemble-averaged differential Mueller matrix elements can be computed from the three backscattering coefficients $\sigma_{v v}^{0}$, $\sigma_{h h}^{0}, \sigma_{v h}^{0}$ and the two phase-difference parameters $\alpha, \zeta$ as follows:

$$
\begin{aligned}
& M_{11}^{0}=\frac{1}{4 \pi} \sigma_{v v}^{0} \\
& M_{22}^{0}=\frac{1}{4 \pi} \sigma_{h h}^{0} \\
& M_{12}^{0}=M_{21}^{0}=\frac{1}{4 \pi} \sigma_{v h}^{0} \\
& M_{33}^{0}=\frac{1}{4 \pi}\left(\alpha \cos \zeta \sqrt{\sigma_{v v}^{0} \sigma_{h h}^{0}}+\sigma_{v h}^{0}\right) \\
& M_{44}^{0}=\frac{1}{4 \pi}\left(\alpha \cos \zeta \sqrt{\sigma_{v v}^{0} \sigma_{h h}^{0}}-\sigma_{v h}^{0}\right) \\
& M_{43}^{0}=-M_{34}^{0}=\frac{1}{4 \pi} \alpha \sin \zeta \sqrt{\sigma_{v v}^{0} \sigma_{h h}^{0}} .
\end{aligned}
$$

\section{Polarimetric EXPERIMENTAL ObSERVATIONS}

In support of the model development, an extensive database was generated of the $v v-, h h$-, $v h$-polarized backscattering coefficients, the degree of correlation and the co-polarized phasedifference, obtained by a combination of ground-based scatterometers and the JPL airborne SAR system over a wide variety of bare soil surfaces. This database also includes precise

$$
\bar{M}^{0}=\left[\begin{array}{cccc}
\left\langle\left|S_{v v}^{0}\right|^{2}\right\rangle & \left\langle\left|S_{v h}^{0}\right|^{2}\right\rangle & 0 & 0 \\
\left\langle\left|S_{v h}^{0}\right|^{2}\right\rangle & \left\langle\left|S_{h h}^{0}\right|^{2}\right\rangle & 0 & 0 \\
0 & 0 & \left\langle\operatorname{Re}\left(S_{v v}^{0} S_{h h}^{0 *}\right)\right\rangle+\left\langle\left|S_{v h}^{0}\right|^{2}\right\rangle & -\left\langle\operatorname{Im}\left(S_{v v}^{0} S_{h h}^{0 *}\right)\right\rangle \\
0 & 0 & \left\langle\operatorname{Im}\left(S_{v v}^{0} S_{h h}^{0 *}\right)\right\rangle & \left\langle\operatorname{Re}\left(S_{v v}^{0} S_{h h}^{0 *}\right)\right\rangle-\left\langle\left|S_{v h}^{0}\right|^{2}\right\rangle
\end{array}\right]
$$


ground truth data such as the surface roughness parameters and the volumetric soil moisture contents for all soil surfaces. The surface roughness for each field was measured by averaging 1-m profiles collected by a laser profilometer and $3.5-\mathrm{m}$ profiles acquired using chart paper and spray paint The soil moisture content was measured by a dielectric probe and by soil sampling. Most of the soil surfaces were agricultural fields and their soil types were primarily silt loam, loam, or sandy loam. The database includes the following seven polarimetric measurements:

\section{A. LCX POLARSCAT Data-1}

This data set was obtained by a truck-mounted polarimetric scatterometer at $1.5 \mathrm{GHz}, 4.75 \mathrm{GHz}$ and $9.5 \mathrm{GHz}$ at the Botanical Garden of the University of Michigan in 1990 [6], [8]. Four different, random, bare soil fields were generated and each was measured under two different moisture conditions at incidence angles ranging from $10^{\circ}$ to $70^{\circ}$. For each data point (different frequency, polarization configuration, incidence angle, roughness and moisture content), more than 60 independent samples were taken to achieve good statistical representation of the measured backscatter. A summary of the scatterometer characteristics and the surface roughness and soil moisture data for these soil fields is given in [6].

\section{B. POLARSCAT Data-2}

This data set was obtained by a truck-mounted polarimetric scatterometer at $1.25 \mathrm{GHz}$ and $5.3 \mathrm{GHz}$ during the cross-calibration experiment of the JPL AirSAR and by a truck-mounted polarimetric scatterometer at Pellston, Michigan in 1991 [17]. Three different rough soil fields $(300 \mathrm{~m} \times 100 \mathrm{~m})$ were generated before the experiment and measured at incidence angles of $30^{\circ}, 40^{\circ}$, and $50^{\circ}$. The roughness parameters and soil moisture contents of the surfaces are given in [17].

\section{POLARSCAT Data-3}

Four different soil surfaces were generated by flattening, tilling, raking and plowing four different agricultural fields located near Ypsilanti, Michigan. Then, the backscattering coefficients of these four fields were measured by a truck-mounted polarimetric scatterometer at $1.25 \mathrm{GHz}, 5.3 \mathrm{GHz}$, and $9.6 \mathrm{GHz}$ at incidence angles of $20^{\circ}, 30^{\circ}, 40^{\circ}, 50^{\circ}, 60^{\circ}$, and $70^{\circ}$ over a period of two months. During this period, the surfaces underwent several drying and wetting cycles and each surface was measured under eight different soil moisture conditions. Table I provides a summary of the associated surface roughness and soil moisture conditions.

\section{Polarimetric Scatterometer Data-4}

An indoor polarimetric scatterometer system was used to measure the backscattering coefficients of two bare soil surfaces at $15 \mathrm{GHz}$ at incidence angles ranging from $0^{\circ}$ to $70^{\circ}$ at Hong-ik University, Seoul, Korea, in 1999. The surface parameters measured for the first surface were $s=0.45 \mathrm{~cm}$, $l=6.89 \mathrm{~cm}$ and $m_{v}=0.09$ and those for the second surface were $s=0.40 \mathrm{~cm}, l=5.65$ and $m_{v}=0.19$.
TABLE I

MEASURED SOIL-SURFACE PARAMETERS FOR POLARSCAT DATA-3

\begin{tabular}{|c|c|c|c|c|c|}
\hline \multicolumn{2}{|c|}{ Surface Parameters } & S-1 & S-2 & S-3 & S-4 \\
\hline \multicolumn{2}{|c|}{ rms height, $s(\mathrm{~cm})$} & 0.55 & 0.94 & 1.78 & 3.47 \\
\hline \multicolumn{2}{|c|}{ Correl. length, ${ }^{l}(\mathrm{~cm})$} & 9.4 & 6.9 & 8.3 & 11.0 \\
\hline \multirow{8}{*}{$\begin{array}{l}\text { Volumetric } \\
\text { soil moisture } \\
\text { content } \\
\text { (top } 3 \mathrm{~cm} \\
\text { layer) }\end{array}$} & M1 & 0.091 & 0.091 & 0.070 & 0.070 \\
\hline & M2 & 0.069 & 0.069 & 0.076 & 0.060 \\
\hline & M3 & 0.073 & 0.043 & 0.045 & 0.045 \\
\hline & M4 & 0.146 & 0.146 & 0.243 & 0.243 \\
\hline & M5 & 0.074 & 0.087 & 0.059 & 0.100 \\
\hline & M6 & 0.121 & 0.121 & 0.086 & 0.086 \\
\hline & M7 & - & - & 0.291 & 0.291 \\
\hline & M8 & 0.262 & - & 0.283 & - \\
\hline
\end{tabular}

\section{E. AirSAR Data-1}

During the cross-calibration experiment involving the JPL AirSAR and a truck-mounted polarimetric scatterometer at Pellston, Michigan in 1991 [17], the backscatter responses at $1.25 \mathrm{GHz}$ and $5.3 \mathrm{GHz}$ were measured by the SAR at three different incidence angles (about $25^{\circ}, 35^{\circ}$ and $55^{\circ}$ ) for the same soil surfaces observed by the POLARSCAT Data-2.

\section{F. AirSAR Data-2}

During the Washita '92 experiment conducted at Chichasha, Oklahoma in 1992, the JPL AirSAR obtained backscatter data from various fallow fields for two weeks while several working groups were collecting ground truth data. Backscatter data for a large bare soil field (approximately $700 \mathrm{~m} \times 1400 \mathrm{~m}$ ) are available for various soil moisture conditions at $1.25 \mathrm{GHz}$ and $5.3 \mathrm{GHz}$. The recorded soil surface parameters are $s=1.82 \mathrm{~cm}$, $l=17.75 \mathrm{~cm}$ and $m_{v}=0.287,0.224,0.241,0.181,0.136$, and 0.116 at about $55^{\circ}$ and $m_{v}=0.241,0.175$ at about $34^{\circ}$.

\section{G. AirSAR Data-3}

In 1993, the JPL AirSAR was used to measure the radar backscatter response of various agricultural fields near Davis, CA. Backscatter and ground surface data were recorded for 19 bare soil fields at $1.25 \mathrm{GHz}$. The rms heights of those fields ranged from $0.61 \mathrm{~cm}$ to $2.5 \mathrm{~cm}$ and the correlation lengths varied from $1.73 \mathrm{~cm}$ to $11.9 \mathrm{~cm}$ and the volumetric moisture contents varied from 0.035 to 0.144 .

A summary of dynamic range for soil parameters $m_{v}, k s$, and $k l$ is given in Table II.

\section{Modeling Procedure and Verification}

The input parameters for the intended polarimetric model include incidence angle $\theta$, the volumetric soil moisture content $m_{v}$ and the roughness parameters $k s$ and $k l$, where $s$ is the rms height, $l$ is the correlation length and $k$ is the wavenumber. The surface roughness spectrum was also considered in the modeling procedure by comparing various roughness spectra [18] with the measured radar backscatter. Because the backscatter is only weakly dependent on soil type, in comparison with its response to surface roughness and soil moisture, the soil type has 
TABLE II

DYNAMIC RANGE OF SOIL PARAMETERS

\begin{tabular}{c|c|c|c|c}
\hline \multirow{2}{*}{$\begin{array}{c}\text { Range } \\
\text { Parameter }\end{array}$} & \multicolumn{2}{|c|}{$90 \%$ range } & \multicolumn{2}{c}{$95 \%$ range } \\
\cline { 2 - 5 } & Min. & Max. & Min. & Max. \\
\hline$m_{v}\left(\mathrm{~cm}^{3} / \mathrm{cm}^{3}\right)$ & 0.043 & 0.283 & 0.040 & 0.291 \\
\hline$k s(s:$ rms height $)$ & 0.14 & 6.00 & 0.13 & 6.98 \\
\hline$k l(l:$ cor. length $)$ & 1.81 & 21.65 & 1.67 & 22.12 \\
\hline$s / l$ & 0.059 & 0.387 & 0.048 & 0.388 \\
\hline
\end{tabular}

been excluded in this model. The soil moisture content $m_{v}$ is used in the model instead of the complex dielectric constant for simplicity. Moreover, the soil moisture content of the top 3-cm soil-surface layer is used at all frequencies because it was shown that the top $2 \sim 3 \mathrm{~cm}$ soil layer exhibits the greatest influence on the radar backscatter response even though the wave may penetrate deeper into the soil for a dry surface at L-band [19].

\section{A. vh-Polarized Backscattering Coefficient}

It was found that the cross-polarized backscattering coefficient of the semi-empirical model described in [6] agrees very well with the measurements, especially with regard to its dependence on $\theta$. The model expresses $\sigma_{v h}^{0}$ in terms of the Fresnel reflectivity (or indirectly through the complex dielectric constant), the incidence angle and the roughness parameter $k s$. For a typical agricultural soil, such as silt loam or sandy loam, the Fresnel reflectivity exhibits an approximately linear dependence on the volumetric soil moisture content [20]. At nadir the relationship assumes the approximate form $\Gamma_{0}=m_{v}^{0.7}$ and is valid over the soil moisture range of $0.03<m_{v}<0.35$.

After examining the angular patterns of the measured data, we selected $(\cos \theta)^{c}$ as a candidate function for characterizing the angular dependence and $1-\exp \left[-a(k s)^{b}\right]$ function is used to account for the response to surface roughness. This roughness function satisfies the conditions that the cross-polarized backscattering coefficient approaches zero for a smooth (near flat) surface $(k s \rightarrow 0)$ and that on the other extreme it becomes independent of $k s$ for a very rough surface $(k s \rightarrow \infty)$. Hence, an overall functional form for the cross-polarized backscattering coefficient is proposed as follows:

$$
\sigma_{v h}^{0}=a m_{v}^{b}(\cos \theta)^{c}\left[1-\exp \left(-d(k s)^{e}\right)\right] .
$$

The magnitudes of constants $a, b, c, d$ and $e$ were determined through data fitting, using the database, by applying the minimum mean square error (MMSE) technique. The process led to the following values $a=0.11, b=0.7, c=2.2, d=0.32$, and $e=1.8$.

The data fitting has been performed using all data listed in the previous section. Table III shows the root-mean-square errors obtained from the data-fit process.

Fig. 3 shows a comparison between our model and the IEM for an incidence angle of $45^{\circ}$ and a soil moisture content $m_{v}=$ 0.13 . The figure also includes data measured at angles in the range $35^{\circ}<\theta<55^{\circ}$ and for moisture in the range $0.03<$ $m_{v}<0.3$. For the IEM computation, an approximate backscattering model for surfaces with small and moderate rms heights
TABLE III

RoOT-MEAN-SQuare ERrors For THE DATA-FITTING Process

\begin{tabular}{c|c|c|c|c}
\hline & \multirow{2}{*}{$\begin{array}{c}\text { rms } \\
\text { error }\end{array}$} & \multicolumn{2}{|c|}{ Data range (95\%) } & Number \\
\cline { 3 - 4 } & & Min. & Max. & of points \\
\hline$\sigma_{v h}^{0}(\mathrm{~dB})$ & 2.35 & -44.35 & -14.94 & 651 \\
\hline$q(\mathrm{~dB})$ & 2.06 & -22.03 & -8.49 & 651 \\
\hline$p(\mathrm{~dB})$ & 0.82 & -4.90 & +0.93 & 651 \\
\hline$\alpha$ & 0.116 & 0.445 & 1.010 & 637 \\
\hline$\zeta($ deg. $)$ & 9.4 & 1.2 & 49.4 & 623 \\
\hline
\end{tabular}

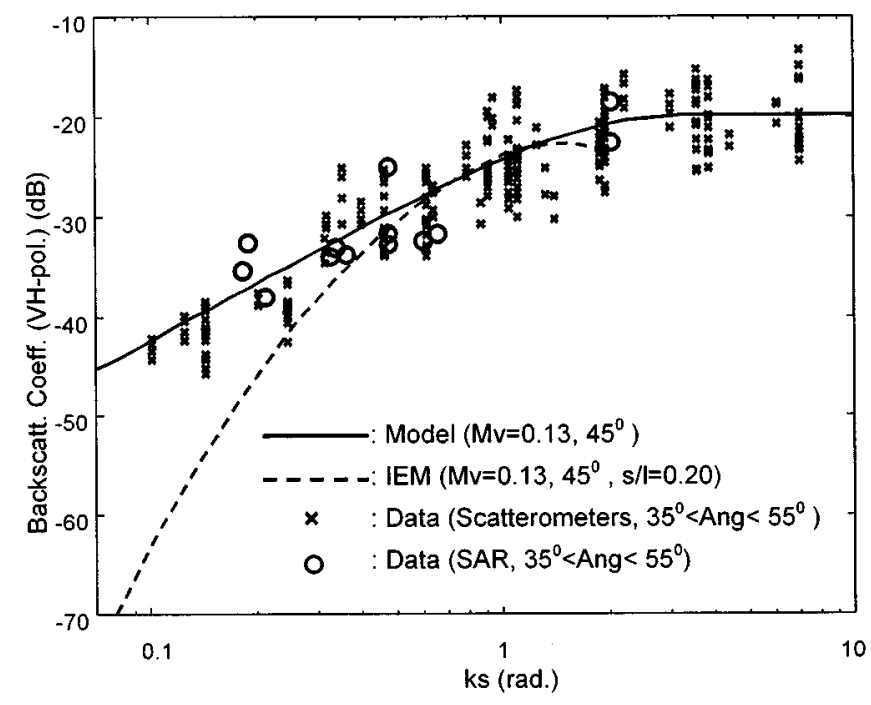

Fig. 3. Measurements of $\sigma_{v h_{h}}^{0}$ for conditions over the range of $35^{\circ}<\theta<55^{\circ}$, $0.03<m_{v}<0.3$ and $0.03<s / l<0.32$. The measurements are compared with curves calculated using the IEM and the semi-empirical model, both for $\theta=45^{\circ}$ and $m_{v}=0.13$.

$(k s<2)[3$, Ch.5] with an exponential correlation function with $k l=k s / 0.2$ was used. Our proposed model agrees well with the measurement across the entire range of $k s$ and agrees with the IEM for the range of $k s>0.4$.

\section{B. vv-Polarized Backscattering Coefficient}

The cross-polarized ratio $q$ is defined as $q \equiv \sigma_{v h}^{0} / \sigma_{v v}^{0}$ as in [6], where it is expressed as proportional to the term $\{1-$ $\exp [-k s]\}$ and to $\sqrt{\Gamma_{0}}$. Analysis of the database shows that the measured values of $q$ agree quite well with $a\left\{1-\exp \left[-b k s^{c}\right]\right\}$. Fig. 4 shows the measured values of $q$ for $0.03<m_{v}<0.3$, $0.03<s / l<0.32$ at $29^{\circ}<\theta<51^{\circ}$, compared with the functional form given by $0.12\left\{1-\exp \left[-0.9(k s)^{0.8}\right]\right\}$. Also shown is a set of curves calculated on the basis of the IEM, assuming an exponential autocorrelation function with $m_{v}=0.20, \theta=40^{\circ}$ and $s / l=0.25$.

Data analysis also shows that the sensitivity of the measured $q$ to incidence angle $\theta$ is high enough for modeling, while that to the soil moisture $m_{v}$ is very weak. We examined the sensitivities of $q$ to $\theta$ and $m_{v}$ using the IEM and confirmed that the $q$ increases rapidly with incidence angles for $0^{\circ} \leq \theta \leq 40^{\circ}$, particularly for a surface with a small surface slope $(s / l=0.05)$, but becomes almost insensitive to the incidence angle at angles 


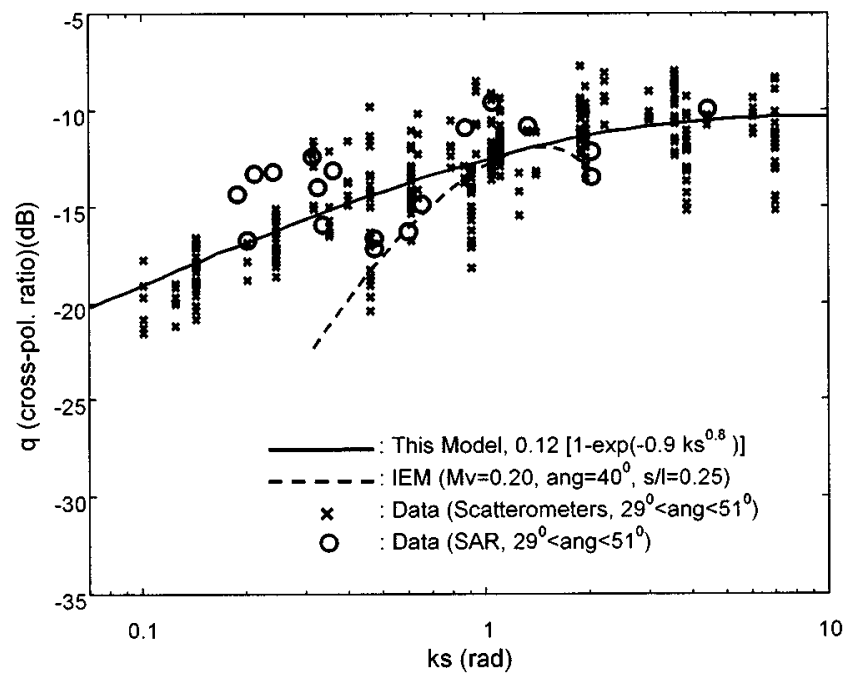

Fig. 4. Measured variation of the cross-polarized ratio $\sigma_{v h^{0}}{ }^{0} / \sigma_{v v}{ }^{0}$ with $k s$, where the measurements cover the range $29^{\circ}<\theta<51^{\circ}, 0.03<m_{v}<0.3$ and $0.03<s / l<0.32$. Curves calculated in accordance with the semi-empirical model and the IEM are shown for comparison.

greater than $40^{\circ}$ and that $q$ is insensitive to soil moisture over the range $0.03<m_{v}<0.35$.

Based on the preceding analysis, we propose the following functional form for the cross-polarized ratio:

$$
q=\frac{\sigma_{v h}^{0}}{\sigma_{v v}^{0}}=a\left(\frac{s}{l}+\sin b \theta\right)^{c}\left\{1-\exp \left[-d(k s)^{e}\right]\right\}
$$

where $a, b, c, d$ and $e$ are constants to be determined by applying MMSE fitting to the database. The best data-fit gave $a=0.10$, $b=1.3, c=1.2, d=0.9$, and $e=0.8$.

Upon combining (12) and (13), we obtain the following expression for the $v v$-polarized backscattering coefficient, see

$$
\begin{aligned}
\sigma_{v v}^{0} & =\frac{\sigma_{v h}^{0}}{q} \\
& =\frac{0.11 m_{v}^{0.7} \cos ^{2.2} \theta\left\{1-\exp \left[-0.32(k s)^{1.8}\right]\right\}}{0.10\left[\frac{s}{l}+\sin (1.3 \theta)\right]^{1.2}\left\{1-\exp \left[-0.9(k s)^{0.8}\right]\right\}} .
\end{aligned}
$$

We note that the dependence of $\sigma_{v v}^{0}$ on the parameter $k s$ can be approximated as $\sigma_{v v}^{0} \propto k s$ for $k s<2$ and $\sigma_{v v}^{0} \propto 1$ (independent of $k s$ ) for $k s>2$. Fig. 5 displays measured values of $\sigma_{v v}^{0}$ as a function of $k s$. Also shown is our semi-empirical model calculated for median conditions, as well as the IEM $(k s<2)$ and GO model ( $k s>3)$. For the GO model computation, a surface correlation function $\rho(\xi)=\left[1+\xi^{2} / l^{2}\right]^{-1.5}$ was assumed [1, Ch.12]. Our empirical model agrees well with the measurements over the entire range of $k s$ and it also agrees with the IEM for $k s<2$ and with the GO model for $k s>3$.

\section{C. hh-Polarized Backscattering Coefficient}

Next, we examined the co-polarized ratio $p \equiv \sigma_{h h}^{0} / \sigma_{v v}^{0}$. The SPM, which is valid for small $k s$ values, shows that the $\sigma_{v v}^{0}$ is higher than $\sigma_{h h}^{0}$ and that the ratio $p$ depends on the dielectric constant (and therefore on the soil moisture content) and the incidence angle, while the GO model, which is valid for large $k s$ values, shows that $\sigma_{v v}^{0}$ is always equal to $\sigma_{h h}^{0}$. We know that

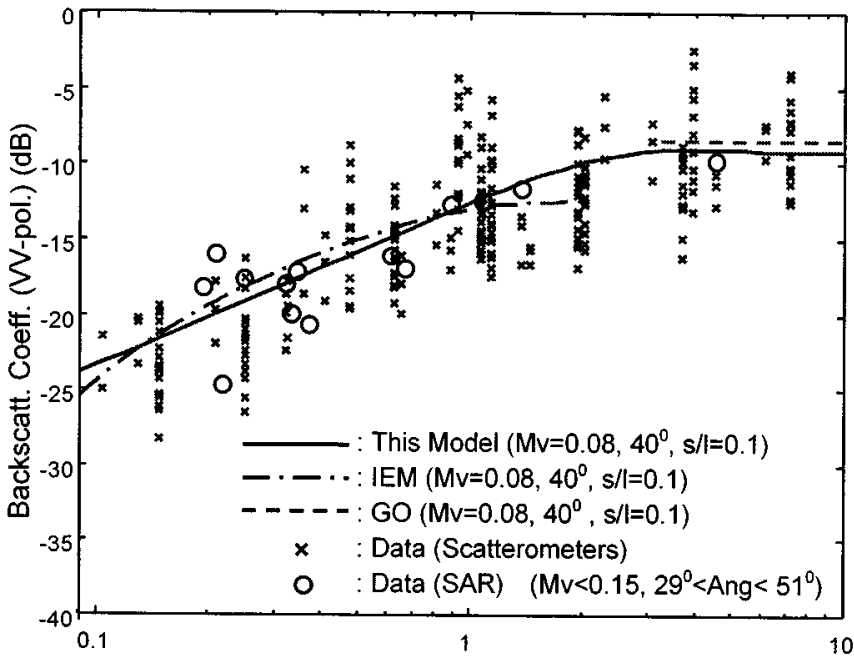

Fig. 5. Plots of $\sigma_{v v}^{0}$, calculated in accordance with the IEM, the GO model and the semi-empirical model, for $\theta=40^{\circ}, m_{v}=0.08$ and $s / l=0.1$. Also included are measurements over the range $29^{\circ}<\theta<51^{\circ}, 0.03<m_{v}<$ 0.15 and $0.03<s / l<0.32$.

$p=1(0 \mathrm{~dB})$ at normal incidence $\left(\theta=0^{0}\right)$ for any surface and it is also equal to 1 when the surface is very rough $(k s \rightarrow \infty)$ at any incidence angle. Incorporating these limiting cases with expectations on the variations of $p$ with $m_{v}$ and $k s$, we propose the following functional form:

$$
p \equiv \frac{\sigma_{h h}^{0}}{\sigma_{v v}^{0}}=1-\left(\frac{\theta}{90^{\circ}}\right)^{a m_{v}^{-b}} \cdot e^{-c(k s)^{d}}
$$

where $\theta$ is the incidence angle in degrees and $a, b, c$ and $d$ are constants to be found from MMSE fitting using the database. This form uses the volumetric soil moisture content $m_{v}$ (for simplicity) instead of the Fresnel reflectivity at nadir [6]. The best data-fit gave $a=0.35, b=0.65, c=0.4$ and $d=1.4$.

Fig. 6 shows the variation of the measured $p$ to $k s$ for data over the range $0.03<m_{v}<0.3$ and $0^{\circ}<\theta<70^{\circ}$. For comparison, model curves are shown for two extreme cases, namely $m_{v}=0.03$ and $\theta=10^{\circ}$ and $m_{v}=0.3$ and $\theta=70^{\circ}$, as well as curves based on the IEM for the same extreme cases.

We can compute the $h h$-polarized backscattering coefficient $\sigma_{h h}{ }^{0}$ by simply combining (14) and (15) or by combining (12), (13) and (15)

$$
\sigma_{h h}^{0}=p \sigma_{v v}^{0}=\frac{p}{q} \sigma_{v h}^{0} .
$$

In Fig. 7 we compare the model-calculated angular responses of $\sigma_{v v}^{0}, \sigma_{h h}^{0}$ and $\sigma_{v h}^{0}$ for a surface with $k s=0.477, k l=4.65$ at about $55^{\circ}$ with backscattering values measured by the JPL AirSAR. Good agreement is observed between the model and the measured data.

\section{Degree of Correlation}

Until the present study, no theoretical model has been published containing explicit expressions for the statistical parameters of the phase-difference PDF, namely the degree of correlation $\alpha$ and the co-polarized phase-difference $\zeta$. Hence, our model effort will rely on polarimetric measurements of bare soil surfaces and on numerical computations of backscattering 


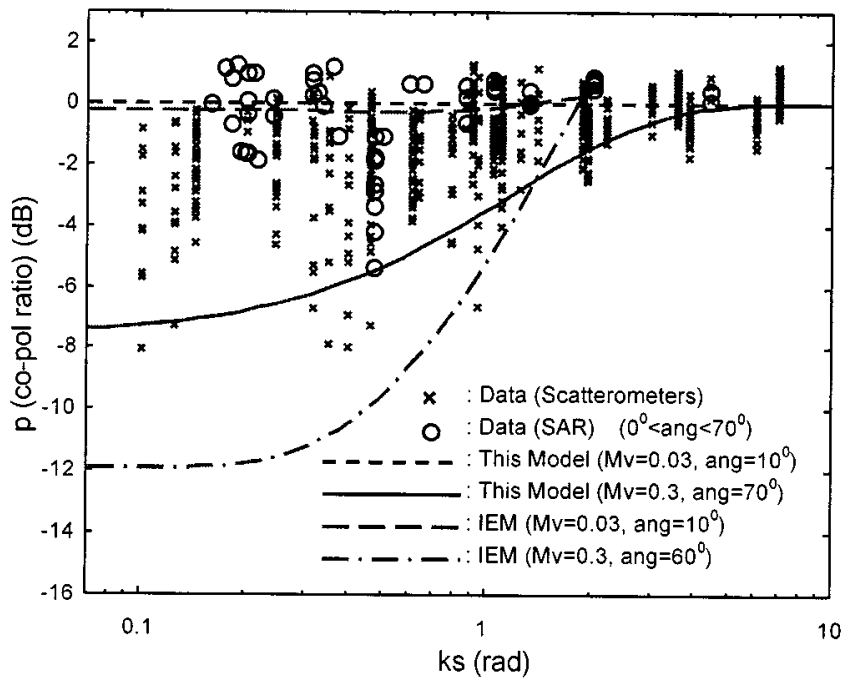

Fig. 6. Variation of the co-polarized ratio $\sigma_{h h_{2}}^{0} / \sigma_{v v}^{0}$ with $k s$ for measurements over the range $0.03<m_{v}<0.3$ and $0^{\circ}<\theta<70^{\circ}$. Curves calculated on the basis of the semi-empirical model, the IEM and the GO model, are shown for two relatively extreme surface conditions.

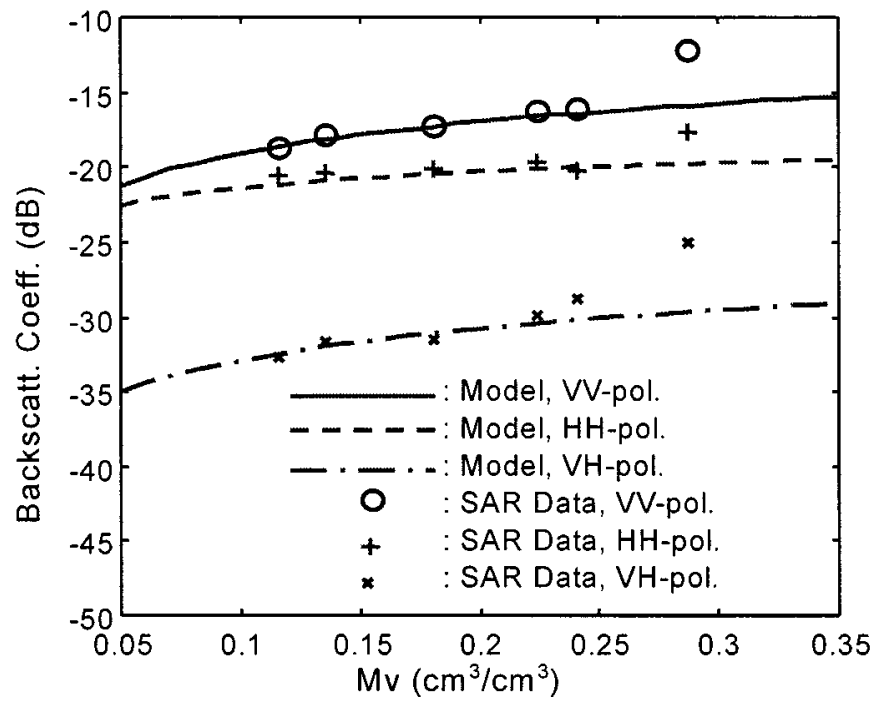

Fig. 7. Polarimetric semi-empirical model compared with SAR data for a surface with $k s=0.477, k l=4.65$ at $54.7^{\circ}<\theta<57.0^{\circ}: \sigma_{v \vartheta}^{0}, \sigma_{h h_{l}}^{0}$ and $\sigma_{v h_{t}}^{0}$ versus $m_{v}$.

from one-dimensional inhomogeneous, dielectric, rough surfaces [21]. For an isotropic homogeneous rough surface, there is no phase-difference between the $v v$ - and $h h$-polarized backscattering amplitudes at $\theta=0^{\circ}$. Therefore, the PDF of the phasedifference angle is a delta function at $\theta=0^{\circ}$, which corresponds to $\alpha=1$ (Fig. 2). The measurement database and the numerical computation show that $\alpha$ decreases as $\theta$ increases and the angular variation of $\alpha$ depends strongly on the surface roughness and weakly on the soil moisture content.

After close examination of the database, the following functional form was chosen:

$$
\alpha=1-A(\sin \theta)^{B}
$$

where $A$ and $B$ may depend on the surface roughness and soil moisture content. At first, $A$ and $B$ were determined for each

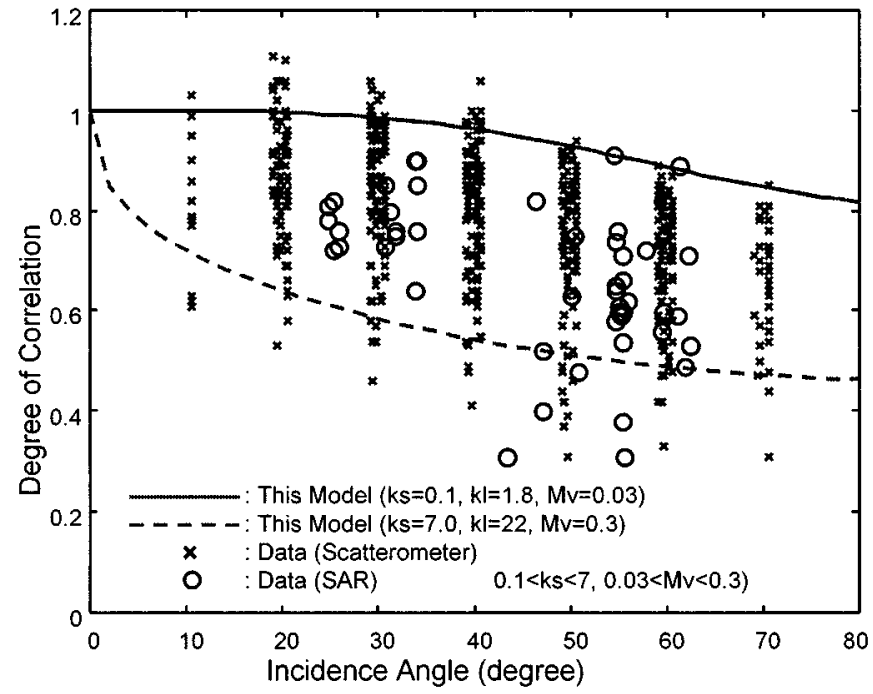

Fig. 8. Measured degree of correlation compared with model predictions for two relatively extreme surface conditions.

of many subsets in the database, with each subset defined over a narrow range of the parameters $m_{v}, k s, k l$ and $s / l$. Then, functional forms were deployed for $A$ and $B$ leading to

$$
\alpha=1-\left(a+b k l+c m_{v}\right) \cdot(\sin \theta)^{d(k s)^{-\epsilon}} .
$$

Application of MMSE data-fit to the measurement database provided the following values for the constants: $a=0.17, b=$ $0.01, c=0.5, d=1.1$ and $e=0.4$. Fig. 8 shows the variation of the measured $\alpha$ with $\theta$ for the range $0.03<m_{v}<0.3$, $0.1<k s<7$ and $1.8<k l<22$ and the two calculated curves bounding the data represent two relatively extreme conditions.

\section{E. Co-Polarized Phase-Difference}

The co-polarized phase-difference $\zeta$ is a measure of the mean value of the co-polarized phase angle $\phi_{c}$. At $\theta=0^{\circ}, \zeta$ should be zero because there should be no phase-difference between the $v v$ - and $h h$-polarized backscattering amplitudes for an isotropic surface. A data-fitting process similar to that used in the preceding section led to the following functional dependence on the surface parameters:

$$
\zeta=\left(a+b m_{v}-c \frac{s}{l}\right) \theta
$$

where $\theta$ is the incidence angle in degrees. The MMSE data-fit to the measurement database provided $a=0.44, b=0.95$ and $c=1.0$. In Fig. 9 we compare model calculation of the co-polarized phase-difference $\zeta$ with all of the measured data.

As shown in Fig. 9, the data measured by the AirSAR are about $15^{\circ}$ lower than those measured by the polarimetric scatterometers. The phase-difference parameters measured by the polarimetric scatterometers are accurate because the data were calibrated by the differential Mueller matrix technique using the polarimetric response of a calibration target over the entire mainlobe of the scatterometer [15]. When a traditional calibration technique for a distributed target is used, i.e., the differential Mueller matrix is approximated by the Mueller matrix divided simply by an illuminated area, the $\alpha$ and the $\zeta$ are inaccurate as shown in [15]. For the scatterometer data, the $\alpha$ values 


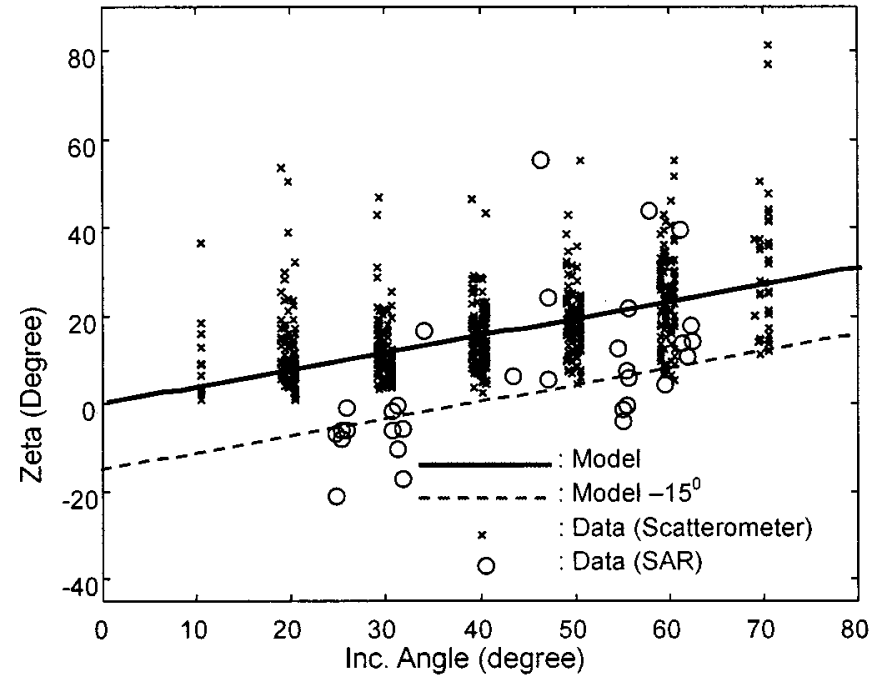

Fig. 9. Measured co-polarized-phase-difference compared with model predictions for median soil parameters.

obtained by the old illumination-integral calibration technique were about 0.8 times the $\alpha$ values determined by the new accurate differential Muller matrix calibration technique. It should be noted that the degree of correlation measured by the JPL AirSAR fits better to the model (17) multiplied by a factor of $0.8 \sim 0.85$.

Table IV shows maximum sensitivities of the models for $\sigma_{v h}^{0}$, $p, q, \alpha$ and $\zeta$ over the $95 \%$ ranges of the soil parameters $k s, m_{v}$, $k l, s / l$ (Table II), as well as over the range of $\theta\left(10^{\circ} \leq \theta \leq 70^{\circ}\right)$.

\section{F. Differential Mueller Matrix}

As described in Section I, the differential Mueller matrix can be computed from $\sigma_{v v}^{0}, \sigma_{h h}^{0}, \sigma_{v h}^{0}, \alpha$ and $\zeta$, each of which can be computed using the expressions provided by the semi-empirical polarimetric model in terms of the surface parameters. Comparison between the model and measured data can be made in terms of the Mueller matrix. At $\theta=30^{\circ}$, a surface with $k s=0.126$, $k l=2.62$, and $m_{v}=0.126$ was measured by the scatterometer to have a differential Mueller matrix

$$
\overline{\bar{M}}_{\text {meas }}=\left[\begin{array}{cccc}
0.0012 & 0.00002 & 0.0000 & 0.0000 \\
0.00002 & 0.0008 & 0.0000 & 0.0000 \\
0.0000 & 0.0000 & 0.0010 & -0.0002 \\
0.0000 & 0.0000 & 0.0002 & 0.0010
\end{array}\right]
$$

For the surface parameters associated with the measured matrix given in (19), such a process leads to

$$
\overline{\bar{M}}_{\text {comp }}=\left[\begin{array}{cccc}
0.0012 & 0.00001 & 0.0000 & 0.0000 \\
0.00001 & 0.0009 & 0.0000 & 0.0000 \\
0.0000 & 0.0000 & 0.0009 & -0.0003 \\
0.0000 & 0.0000 & 0.0003 & 0.0009
\end{array}\right] \text {. }
$$

The corresponding elements of the two differential Mueller matrices are in very good agreement. We note that elements other than $M 11, M 22, M 33, M 34, M 43$ and $M 44$ of the differential Mueller matrix are negligible for radar backscattering from soil surfaces.
TABLE IV

MaXimum Sensitivity of THE MOdel on Each PaRAmeter IN ITS

\begin{tabular}{|c|c|c|}
\hline Model & $\begin{array}{l}\text { Para- } \\
\text { meter }\end{array}$ & Maximum Sensitivity \\
\hline \multirow{3}{*}{$\sigma_{v h}^{0}$} & $k s$ & $20.9 \mathrm{~dB}$ \\
\hline & $\theta$ & $10.3 \mathrm{~dB}$ \\
\hline & $m_{v}$ & $6.0 \mathrm{~dB}$ \\
\hline \multirow{3}{*}{$p$} & $k s$ & $\begin{array}{l}7.1 \mathrm{~dB}\left(\theta=70^{\circ}, m_{v}=0.29\right) \\
0 \mathrm{~dB}\left(\theta=10^{0}, \quad m_{v}=0.04\right)\end{array}$ \\
\hline & $\theta$ & $\begin{array}{l}6.2 \mathrm{~dB}\left(k s=0.13, \quad m_{v}=0.29\right) \\
0 \mathrm{~dB}\left(k s=6.98, \quad m_{v}=0.04\right)\end{array}$ \\
\hline & $m_{v}$ & $\begin{array}{l}4.1 \mathrm{~dB}\left(k s=0.13, \theta=70^{0}\right) \\
0 \mathrm{~dB}\left(k s=6.98, \theta=10^{\circ}\right)\end{array}$ \\
\hline \multirow{3}{*}{$q$} & $k s$ & $7.9 \mathrm{~dB}$ \\
\hline & $\theta$ & $\begin{array}{l}7.0 \mathrm{~dB}(s / l=0.048) \\
4.3 \mathrm{~dB}(s / l=0.388)\end{array}$ \\
\hline & $s / l$ & $\begin{array}{l}4.2 \mathrm{~dB}\left(\theta=70^{\circ}\right) \\
1.5 \mathrm{~dB}\left(\theta=10^{\circ}\right)\end{array}$ \\
\hline \multirow{4}{*}{$\alpha$} & $\theta$ & $\begin{array}{lll}0.453(k s=0.13, & k l=22.1, & \left.m_{v}=0.29\right) \\
0.115(k s=6.98, & k l=1.67, & \left.m_{v}=0.04\right)\end{array}$ \\
\hline & $k s$ & $\begin{array}{lll}0.285\left(\theta=27^{0},\right. & k l=22.1, & \left.m_{v}=0.29\right) \\
0.023\left(\theta=70^{\circ},\right. & k l=1.67, & \left.m_{v}=0.04\right)\end{array}$ \\
\hline & $k l$ & $\begin{array}{ll}0.198(\theta=70, & k s=6.98) \\
0.003(\theta=10, & k s=0.13)\end{array}$ \\
\hline & $m_{v}$ & $\begin{array}{ll}0.122\left(\theta=70^{\circ},\right. & k s=6.98) \\
0.020\left(\theta=10^{\circ},\right. & k s=0.13)\end{array}$ \\
\hline \multirow{3}{*}{$\zeta$} & $\theta$ & $\begin{array}{l}40.1^{0}\left(m_{v}=0.29, s / l=0.048\right) \\
5.4^{0}\left(m_{v}=0.04, s / l=0.388\right)\end{array}$ \\
\hline & $s / l$ & $23.8^{0}\left(\theta=70^{\circ}\right), \quad 3.4^{0}\left(\theta=10^{\circ}\right)$ \\
\hline & $m_{v}$ & $16.7^{0}\left(\theta=70^{0}\right), \quad 2.4^{0}\left(\theta=10^{0}\right)$ \\
\hline
\end{tabular}
95\% RANGE

\section{CONCLUdING REMARKS}

A semi-empirical polarimetric backscattering model was developed for random bare soil surfaces using a combination of truck-mounted scatterometer measurements and airborne SAR observations, both supported by extensive ground observation of the soil surface statistics and moisture content. The functional form of the model was constrained to insure that its predictions are consistent with known theoretical values, such as $\sigma_{v v}^{0}=\sigma_{h h}^{0}$ at normal incidence, $\sigma_{v v}^{0}=\sigma_{h h}^{0}$ for an electromagnetically very rough surface and $\sigma_{v h}^{0} / \sigma_{v v}^{0}$ approaches a constant as surface roughness exceeds $k s=3$. The two distinguishing features of the model is that it not only agrees with experimental observations over a wide range of soil surface conditions, but it also agrees with the IEM and geometrical optics model over their individual regions of validity, thereby encompassing the full range of surface roughness encountered under natural conditions.

\section{ACKNOWLEDGMENT}

The authors would like to thank the reviewers for their useful comments.

\section{REFERENCES}

[1] F. T. Ulaby, M. K. Moore, and A. K. Fung, Microwave Remote Sensing, Active and Passive. Norwood, MA: Artech, 1982, vol. 2. 
[2] A. K. Fung, Z. Li, and K. S. Chen, "Backscattering from a randomly rough dielectric surface," IEEE Trans. Geosci. Remote Sensing, vol. 30, pp. 356-369, Mar. 1992.

[3] A. K. Fung, Microwave Scattering and Emission Models and Their Applications. Boston, MA: Artech, 1994.

[4] C. Hsieh, A. K. Fung, G. N. Nesti, A. J. Sieber, and P. Coppo, "A further study of the IEM surface scattering model," IEEE Trans. Geosci. Remote Sensing, vol. 35, pp. 901-909, July 1997.

[5] K. S. Chen, T. Wu, M. Tsay, and A. K. Fung, "A note on the multiple scattering in an IEM model," IEEE Trans. Geosci. Remote Sensing, vol. 38, pp. 249-256, Jan. 2000.

[6] Y. Oh, K. Sarabandi, and F. T. Ulaby, "An empirical model and an inversion technique for radar scattering from bare soil surfaces," IEEE Trans. Geosci. Remote Sensing, vol. 30, pp. 370-382, Mar. 1992.

[7] P. C. Dubois, J. van Zyl, and E. T. Engman, "Measuring soil moisture with imaging radar," IEEE Trans. Geosci. Remote Sensing, vol. 33, pp. 916-926, July 1995

[8] K. Sarabandi, Y. Oh, and F. T. Ulaby, "Polarimetric radar measurement of bare soil surfaces at microwave frequencies," in Proc. IEEE Geosci. Remote Sensing Symp., June 1991.

[9] K. Sarabandi, "Derivation of phase statistics of distributed targets from the Mueller matrix," Radio Sci., vol. 27, pp. 553-560, Sept.-Oct. 1992.

[10] F. T. Ulaby, K. Sarabandi, and A. Nashashibi, "Statistical properties of the Mueller matrix of distributed targets," Proc. Inst. Elect. Eng., vol. 139, pp. 136-146, Apr. 1992.

[11] H. Skriver, M. T. Svendson, and A. G. Thompsen, "Multitemporal C- and L-band polarimetric signatures of crops," IEEE Trans. Geosci. Remote Sensing, vol. 37, pp. 2413-2428, Sept. 1999.

[12] Y. Oh, K. Sarabandi, and F. T. Ulaby, "An empirical model for phase difference statistics of rough," in Proc. IEEE Geosci. Remote Sensing Symp., vol. III, Aug. 1993, pp. 1003-1005.

[13] _ "An inversion algorithm for retrieving soil moisture and surface roughness from polarimetric radar observation," in Proc. IEEE Geosci. Remote Sensing Symp, vol. III, Aug. 1994, pp. 1582-1584.

[14] F. T. Ulaby and C. Elachi, Radar Polarimetry for Geoscience Applications. Norwood, MA: Artech, 1990.

[15] K. Sarabandi, Y. Oh, and F. T. Ulaby, "Measurement and calibration of differential Mueller matrix of distributed targets," IEEE Trans. Antenna Propagat., vol. 40, pp. 1524-1532, Dec. 1992.

[16] C. Oliver and S. Quegan, Understanding Synthetic Aperture Radar Images. Norwood, MA: Artech, 1998

[17] K. Sarabandi, L. Pierce, Y. Oh, M. C. Dobson, F. T. Ulaby, A. Freeman, and P. Dubois, "Cross-calibration experiment of JPL AIRSAR and truck-mounted polarimetric scatterometer," IEEE Trans. Geosci. Remote Sensing, vol. 32, pp. 975-985, Sept. 1994.

[18] Y. Oh and Y. C. Kay, "Condition for precise measurement of soil surface roughness," IEEE Trans. Geosci. Remote Sensing, vol. 36, pp. 691-695, Mar. 1998.

[19] Y. Oh, "Retrieval of the effective soil moisture contents as a ground truth from natural soil surfaces," in Int. Geosci. Remote Sensing Symp. (IGARSS2000), 2000.

[20] Y. Du, F. T. Ulaby, and M. C. Dobson, "Sensitivity to soil moisture by active and passive microwave sensors," IEEE Trans. Geosci. Remote Sensing, vol. 38, pp. 105-113, Jan. 2000.

[21] K. Sarabandi, Y. Oh, and F. T. Ulaby, "A numerical simulation of scattering from one-dimensional inhomogeneous dielectric random surfaces," IEEE Trans. Geosci. Remote Sensing, vol. 34, pp. 425-432, Mar. 1996.

Yisok Oh (S'88-M'94) received the B.S. degree in electrical engineering from Yonsei University, Seoul, Korea, in 1982, the M.S. degree in electrical and computer engineering from the University of Missouri, Rolla, in 1988, and the Ph.D degree in electrical engineering from the University of Michigan, Ann Arbor, in 1993.

He is currently an Associate Professor in the Department of Electronics and Electrical Engineering, Hong-ik University, Seoul. His current research interests include radar backscatter modeling, radar systems, antennas, and radio wave propagation.
Kamal Sarabandi (S'87-M'90-SM'92-F'00) received the B.S. degree in electrical engineering from Sharif University of Technology, Tehran, Iran, in 1980, and the M.S. degree in mathematics and the Ph.D. degree in electrical engineering from The University of Michigan, Ann Arbor,in 1989.

$\mathrm{He}$ is the Director of the Radiation Laboratory and a Professor in the Department of Electrical Engineering and Computer Science, University of Michigan. His research areas of interest include electromagnetic wave propagation, antennas and microwave, and millimeter-wave radar remote sensing. He has served as the Principal Investigator on many projects sponsored by NASA, JPL, ARO, ONR, ARL, NSF, DARPA, and numerous industries. He has published many book chapters and more than 90 papers in refereed journals on electromagnetic scattering, random media modeling, wave propagation, antennas, microwave measurement techniques, radar calibration, inverse scattering problems, and microwave sensors. He has also had more than 170 papers and invited presentations in national and international conferences and symposia on similar subjects.

Dr. Sarabandi received the prestigious Henry Russel Award from the Regent of The University of Michigan, the GAAC Distinguished Lecturer Award from the German Federal Ministry for Education, Science and Technology, in 1999, and the 1996 Teaching Excellence Award from the EECS Department, University of Michigan. He is listed in Who's Who in American Men \& Women of Science, Who's Who in America, and Who's Who in Electromagnetics. $\mathrm{He}$ is a member of the IEEE Geoscience and Remote Sensing Society (GRSS) ADCOM, Chairman of the Awards Committee of the IEEE GRSS, and a member of IEEE Technical Activities Board Awards Committee. He is serving as the Associate Editor of the IEEE TRANSACTIONS ON ANTENNAS AND PROPAGATION (AP) and the IEEE Sensors Journal. He is also a member of Commission F, URSI, and of The Electromagnetic Academy.

Fawwaz Ulaby (M'68-SM'74-F'80) received the B.S. degree in physics from the American University of Beirut, Lebanon, in 1964 and the M.S.E.E. and $\mathrm{Ph} . \mathrm{D}$. degrees in electrical engineering from the University of Texas, Austin, in 1966 and 1968, respectively.

$\mathrm{He}$ is the Vice President for Research and Williams Distinguished Professor of Electrical Engineering and Computer Science, University of Michigan, Ann Arbor. His current interests include microwave and millimeter-wave remote sensing, radar systems, and radio wave propagation. He has authored several books and published numerous papers and reports on these subjects.

Dr. Ulaby received the IEEE Geoscience and Remote Sensing Distinguished Achievement Award (1983), the IEEE Centennial Medal (1984), The American Society of Photogrammetry's Presidential Citation for Meritorious Service (1984), the NASA Group Achievement Award (1990), the University of Michigan's Distinguished Faculty Achievement Award (1991), and the IEEE Millennium Medal (2000). He is a member of the National Academy of Engineering and serves on several scientific boards and professional committees, including the editorial board of the PROCEEDINGS OF THE IEEE. He served as President of the IEEE Geoscience and Remote Sensing Society, from 1981 to 1982, Executive Editor of Transactions on Geoscience and Remote Sensing, from 1983 to 1985, and as General Chairman of several international symposia. 